\title{
Alternation of left ventricular load by a continuous-flow left ventricular assist device with a native heart load control system in a chronic heart failure model
}

\author{
Mamoru Arakawa, MD, ${ }^{\mathrm{a}, \mathrm{b}}$ Takashi Nishimura, MD, $\mathrm{PhD},{ }^{\mathrm{c}}$ Yoshiaki Takewa, $\mathrm{MD}, \mathrm{PhD},{ }^{\mathrm{a}}$ \\ Akihide Umeki, MD, PhD, ${ }^{\mathrm{d}}$ Masahiko Ando, MD, PhD,${ }^{\mathrm{d}}$ Hideo Adachi, MD, PhD,${ }^{\mathrm{b}}$ and \\ Eisuke Tatsumi, MD, $\mathrm{PhD}^{\mathrm{a}}$
}

\begin{abstract}
Objective: We previously developed a native heart load control system for a continuous-flow left ventricular assist device and demonstrated that the rotational speed synchronized with the cardiac cycle can alter left ventricular preload and myocardial oxygen consumption. In the present study, we assessed this system in a conscious goat model of chronic heart failure.
\end{abstract}

\begin{abstract}
Methods: Chronic heart failure was induced by coronary microsphere embolization of the left ascending artery and subsequent rapid ventricular pacing in 6 goats. After 4 to 6 weeks of rapid pacing, the goats showed a decreased ejection fraction (from $89.7 \% \pm 3.1 \%$ to $53.3 \% \pm 5.4 \%$ ) measured during sinus rhythm. The assist device was implanted by way of a left thoracotomy, and we examined the effects of the continuous, co-pulse, and counterpulse mode on the end-diastolic volume and stroke work, determined from the left ventricular pressure-volume loops.
\end{abstract}

\begin{abstract}
Results: Significant decreases were found in the end-diastolic volume and stroke work in the counterpulse mode relative to the values observed with $0 \%$ bypass $(63.4 \% \pm 15.2 \%$ and $39.1 \% \pm 18.2 \%$, respectively; $P<.01)$. Furthermore, both increased in the co-pulse mode $(82.1 \% \pm 17.6 \%$ and $68.3 \% \pm 22.2 \% ; P<.01)$ compared with those in the continuous mode $(69.6 \% \pm 15.4 \%$ and $54.6 \% \pm 21.6 \%)$ with $100 \%$ bypass.
\end{abstract}

Conclusions: The system offers the possibility to control the left ventricular load by changing the rotational speed of a continuous-flow assist device in synchronization with the cardiac cycle. This system should provide the most favorable left ventricular loading conditions for recovery of the native heart. (J Thorac Cardiovasc Surg 2014;148:698-704)

Since continuous-flow left ventricular (LV) assist devices (LVADs) were first approved, the number of patients treated with continuous-flow LVADs has been increasing owing to the improved prognosis and fewer complications. ${ }^{1,2}$ Most patients with an implanted LVAD have been on the waiting list for heart transplantation and, thus, have had the LVAD implanted as a bridge to transplantation. However, there is a shortage of donor hearts worldwide,

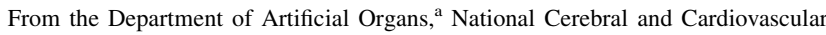
Center Research Institute, Osaka, Japan; Department of Cardiovascular Surgery, ${ }^{b}$ Jichi Medical University Saitama Medical Center, Saitama, Japan; Department of Cardiac Surgery, ${ }^{\mathrm{c}}$ Tokyo Metropolitan Geriatric Hospital, Tokyo, Japan; and

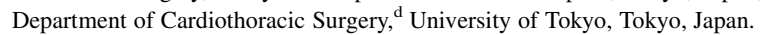

The present study was supported by a grant from Jichi Medical University Graduate School in 2013.

Disclosures: Authors have nothing to disclose with regard to commercial support.

The study was presented at the Annual Conference of the American Society for Artificial Internal Organs, June 15, 2012, San Francisco, Calif.

Received for publication Aug 20, 2013; revisions received Dec 12, 2013; accepted for publication Dec 20, 2013; available ahead of print Feb 9, 2014.

Address for reprints: Mamoru Arakawa, MD, Department of Artificial Organs, National Cardiovascular Center Research Institute, 5-7-1 Fujishiro-dai, Suita, Osaka 565-8565, Japan (E-mail: a_mamoru@mbn.nifty.com).

$0022-5223 / \$ 36.00$

Copyright $($ C 2014 by The American Association for Thoracic Surgery

http://dx.doi.org/10.1016/j.jtcvs.2013.12.049 and this shortage has been especially severe in Japan. ${ }^{3}$ In some cases, myocardial recovery will allow LVAD explantation, and this has been termed "bridge to recovery" (BTR). ${ }^{4,5}$ Because of the extreme shortage of donor hearts, it is important to investigate methods to increase the success rate of BTR. Unloading the left ventricle by an LVAD has been reported to lead to improvement in cardiac function. ${ }^{4}$ In contrast, it has also been reported that excess unloading leads to cardiac atrophy. ${ }^{6}$ We have presumed that a favorable work load is required for cardiac recovery and that the workload should be adapted to the patient's condition.

We have previously reported the development of novel pump control system for a continuous-flow LVAD (EVAHEART; Sun Medical Technology Research Institute, Nagano, Japan). ${ }^{7}$ This system was termed the native heart load control system (NHLCS) and was shown to control the hemodynamic parameters such as pulsatility, ${ }^{8}$ myocardial perfusion, ${ }^{9}$ and LV end-diastolic volume (LVEDV) ${ }^{10}$ by changing the rotational speed (RS) in synchronization with the cardiac cycle.

To evaluate the effects of the NHLCS in detail, it is important to evaluate its long-term performance in a closed-chest animal model with impaired cardiac 


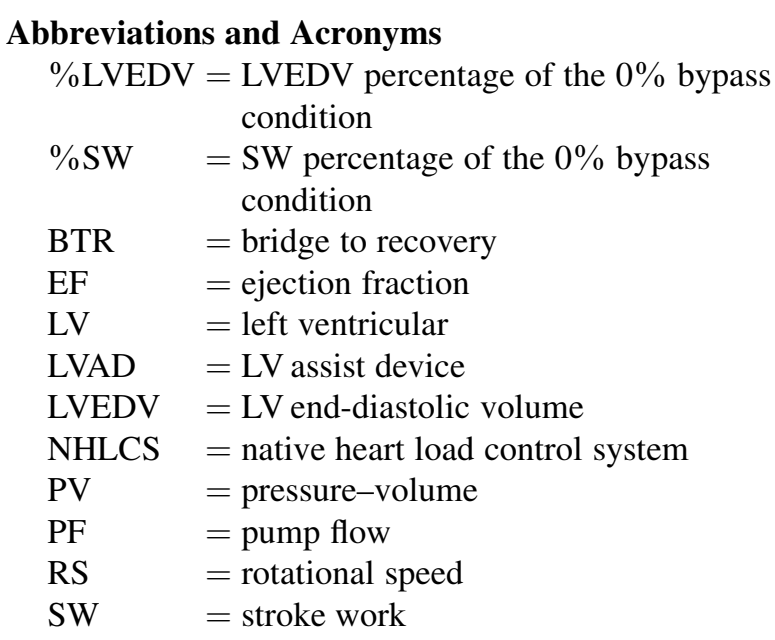

function. ${ }^{11}$ In the present study, we aimed to establish a chronic heart failure model and assess the effect of our EVAHEART on hemodynamics in a conscious animal model of chronic heart failure.

\section{METHODS \\ Animals}

Six adult goats weighing $56.7 \pm 5.1 \mathrm{~kg}$ were maintained in accordance with the guidelines of the Committee on Animal Studies at the National Cerebral and Cardiovascular Center. The National Cerebral and Cardiovascular Center Animal Investigation Committee approved the present study.

\section{Chronic Heart Failure Model}

In the present study, we created chronic heart failure using coronary artery embolization through the left anterior descending coronary artery, as described previously. ${ }^{10,12}$ In brief, a $4 \mathrm{~F}$ Amplatz catheter (Create Medic Co, Ltd, Yokohama, Japan) was inserted through a 4F-long sheath into the left carotid artery toward the left anterior descending coronary artery under fluoroscopic guidance, and $50-\mu \mathrm{m}$ microspheres were injected $(4500 / \mathrm{kg}$ ). We also placed a permanent pacing lead (Tendril STS, $58 \mathrm{~cm}$, St Jude Medical, Inc, St Paul, Minn) into the right ventricle through the right jugular vein and connected it to a pacemaker (TNT-002N, Taisho Biomed Instruments Co, Ltd, Osaka, Japan) that was implanted subcutaneously in the right shoulder. In addition, we placed an arterial pressure line through the right carotid artery. Four days after embolization, rapid ventricular pacing at 200 beats/min was initiated and continued for 4 to 6 weeks. To control the impairment of cardiac function, we measured the ejection fraction (EF) during sinus rhythm using transthoracic echocardiography (Vivid E9, GE Healthcare, Horten, Norway). The duration of pacing was continued until the EF was reduced to $50 \%$ of baseline.

\section{LVAD Implantation}

After 48 hours of fasting, all the goats were sedated with an intramuscular injection of ketamine $(10 \mathrm{mg} / \mathrm{kg})$. General anesthesia was induced and maintained by inhalation of isoflurane ( $1 \%-3 \%$ in oxygen). The goats were fixed in the right recumbent position, intubated, and mechanically ventilated. A left thoracotomy along the fifth costal bone was performed, and pressure lines for aortic pressure and central venous pressure measurement were placed into the left intrathoracic artery and vein, respectively.
This was followed by attachment of an electromagnetic flow meter (EMF-1000, 17-20 mm in diameter, Nihon Kohden, Tokyo, Japan) around the ascending aorta. After heparinization $(200 \mathrm{U} / \mathrm{kg})$, the centrifugal LVAD (EVAHEART; Sun Medical Technology Research Corp, Nagano, Japan) was installed by punching out the LV apex with a $16-\mathrm{mm}$ puncher, inserting the inflow cannula into the left ventricle and suturing the outflow conduit (16-mm J Graft SHIELD NEO, Japan Lifeline, Tokyo, Japan) into the descending aorta. We used an ultrasonic flow meter $(16 \mathrm{~mm}$; TS420, Transonic Systems, Ithaca, NY) to measure the LVAD pump flow (PF). After establishing the pump circuit, we attached 2 ultrasonic crystals (Sonometrics, Ontario, Canada) in the anterior and posterior endocardium to measure the LV diameter in the short axis. This diameter was then used to calculate the LV volume using a single-axis sphere. In addition, a LV pressure line was inserted from the anterior wall.

\section{Study Protocol and LVAD Control}

Two days after LVAD implantation, when the goats had recovered from the acute effects of the operation, we tested 3 drive modes using our previously described NHLCS. ${ }^{10,12}$ With this controller, we were able to change the RS of the EVAHEART for a specific period after the occurrence of an R wave to synchronize it with the cardiac cycles. We defined the systolic phase as $30 \%$ of the RR interval, and the diastolic phase as $65 \%$ of the $\mathrm{RR}$ interval. These parameters were evaluated twice in each goat using the following 3 conditions: (1) the continuous mode (constant RS without the use of the novel controller); (2) the co-pulse mode (increased RS in the systolic phase and decreased RS in the diastolic phase using the novel controller); and (3) the counterpulse mode (decreased RS in the systolic phase and increased RS in the diastolic phase with the novel controller). In these drive modes, we set the RS from 1500 to $3000 \mathrm{rpm}$ to achieve a proper bypass rate. In addition, the difference between the systolic and diastolic phase was set to approximately 500 to $1000 \mathrm{rpm}$.

\section{Statistical Analysis}

The bypass rate was calculated by dividing the pump flow (PF) rate by the sum of the PF and ascending aortic flow rates. We defined full bypass as a bypass rate of $100 \%$. We tested the 3 drive modes at bypass rates of $50 \%$, $75 \%$, and $100 \%$ for 10 minutes. Next, we performed the 3 modes at a bypass rate of $100 \%$ for 12 hours to evaluate the effects of the system for a prolonged period. To evaluate appropriate support, we checked the actual RS and speed changes. The bypass rate was calculated in real time and displayed on a monitor.

The hemodynamic data were recorded using Labchart, version 7 , software (ADInstruments, Castle Hill, Australia). The implanted ultrasonic crystals provided the LV chamber diameter in the short axis, and this was used to estimate the LV volume from a single-axis spherical model. LV pressure-volume (PV) loops were drawn for each mode, and the stroke work (SW) was calculated from the area inside the PV loop using CardioSOFT (Sonometrics). The LV end-diastolic pressure and LVEDV were determined from the PV loops. For the comparison between each control mode, the LVEDV and SW are expressed as percentages of the $0 \%$ bypass condition ( $\% \mathrm{LVEDV}$ and $\% \mathrm{SW}$, respectively).

All numeric data are shown as the average \pm standard deviation. The data between pre-embolization and pre-LVAD implantation were compared using a paired Student's $t$ test. Other comparisons between the groups were performed using repeated measures analysis of variance followed by Tukey's multiple comparison tests. All analyses were performed using the Statistical Package for Social Sciences, version 19 (SPSS Inc, Chicago, Ill).

\section{Effect of LVAD on Hemolysis and Myocardial Histologic Findings}

To evaluate hemolysis, we measured free hemoglobin before and after the 3 modes had been applied for 12 hours in 1 of 6 goats. In addition, an 
in vitro hemolysis test was performed, with mock circulation, according to a previous report. ${ }^{13}$ Fresh bovine blood $(600 \mathrm{~g})$ was used, and the blood temperature was maintained at $37^{\circ} \pm 1^{\circ} \mathrm{C}$. Blood samples were collected for measurement of plasma free hemoglobin just after the pump's condition had become stable and at 30-minute intervals for 4 hours. We drove the EVAHEART in the co-pulse mode at a pulse rate of 60 beats $/ \mathrm{min}$. The RS was set $3000 \mathrm{rpm}$ for high and $1000 \mathrm{rpm}$ for the difference. These settings were representative of the most severe condition of the novel mode. The mean head pressure was set at $100 \mathrm{~mm} \mathrm{Hg}$ and the PF at $5.0 \mathrm{~L} / \mathrm{min}$ for the continuous and co-pulse modes both. These settings were the same as those in a previous report. ${ }^{13}$ We used the normalized index of hemolysis to evaluate hemolysis: normalized index of hemolysis $(\mathrm{g} / 100 \mathrm{~L})=\Delta$ plasma free hemoglobin $(\mathrm{g} / \mathrm{L}) \times$ priming volume (L) $\times([(100-$ hematocrit $) / 100] \times[100 / \mathrm{PF}$ in $\mathrm{L} / \mathrm{min} \times$ time in minutes $])$. We performed biopsy from the apex during LVAD implantation and necropsy when the goats had been killed 1 month after LVAD implantation to evaluate the pathologic changes due to LVAD implantation. The samples were fixed immediately in 10\% neutral-buffered formalin and embedded in paraffin wax. Thin sections were cut and stained with hematoxylin and eosin.

\section{RESULTS}

\section{Chronic Heart Failure Model With LVAD Support}

We performed rapid pacing for $31.4 \pm 5.7$ days. The EF, measured by echocardiography, decreased from $89.7 \% \pm$ $3.1 \%$ at the pre-embolization baseline to $53.3 \% \pm 5.4 \%$ just before LVAD implantation $(P<.01)$.

\section{Hemodynamic and LVAD Data}

Figure 1 shows sample waveforms from the electrocardiogram, aortic pressure, detected $\mathrm{R}$ waves, LV pressure, $\mathrm{PF}$, native flow, and RS. Important differences were present among the PF waveforms with the 3 different modes. The maximum PF increased after an increase in the RS in the systolic phase in the co-pulse mode compared with that in the continuous mode. In contrast, the PF in the diastolic phase increased in the counterpulse mode compared with that in the continuous mode. The hemodynamic data in each mode are listed in Table 1 . The heart rate, central venous pressure, aortic pressure, total flow, and bypass rate were not significantly different among the 3 modes. The average RS in the counterpulse mode decreased significantly compared with those in the continuous and co-pulse modes at each bypass rate.

\section{PV Loops}

Figure 2 shows 1 sample set of PV loops for each mode. The horizontal axis shows the LV volume and the vertical axis the LV pressure. The lower right points indicate the end-diastolic points, and the area inside the loop indicates the SW. The LVEDV (preload) increased in the co-pulse mode and decreased in the counterpulse mode compared with that in the continuous mode.

Figure 3 shows the average LVEDVs measured in the 6 goats. The \%LVEDV and $\% \mathrm{SW}$ were decreased according to the decrease in the bypass rate in the continuous mode. The \%LVEDV was significantly increased in the co-pulse mode compared with that in the continuous mode at the $75 \%$ bypass rate. SW was also increased in the co-pulse mode compared with that in the continuous mode at the $75 \%$ and $100 \%$ bypass rates. Furthermore, the \%LVEDV was significantly increased in the co-pulse mode compared with that in the counterpulse mode at each bypass rate. The $\% \mathrm{SW}$ was also increased in the co-pulse mode compared with that in the counterpulse mode at each bypass rate. In the counterpulse mode, the $\% \mathrm{LVEDV}$ and $\% \mathrm{SW}$ were relatively lower than those in the continuous mode, although the differences were not significant.

\section{Appropriate Support}

To check for appropriate support, we evaluated $7688 \pm$ 400 beats $/ \mathrm{h}$, on average. Appropriate support was achieved for $>90 \%$ of cardiac beats in 1 hour. The most frequent cause of inappropriate support was a failure to sense the $\mathrm{R}$ wave by the controller (4.4\%).

\section{Effect of LVAD on Hemolysis and Myocardial Histologic Findings}

The free hemoglobin in vivo showed a slight elevation after each mode of pumping. The hemoglobin values before and after each mode were 2.7 and $5.2 \mathrm{mg} / \mathrm{dL}$ in the continuous mode, 5.2 to $5.9 \mathrm{mg} / \mathrm{dL}$ in the co-pulse mode, and 4.2 and $6.4 \mathrm{mg} / \mathrm{dL}$ in the counterpulse mode, respectively. Regarding the in vitro hemolysis test, plasma free hemoglobin was slightly increased in the samples collected after the pump had run for 4 hours in the continuous and co-pulse modes both. The hemoglobin values just after the pump's condition had become stable and after 4 hours of pumping in each mode were 0.6 and $3.4 \mathrm{mg} / \mathrm{dL}$ in the continuous mode, 3.4 to $5.0 \mathrm{mg} / \mathrm{dL}$ in the co-pulse mode, and 4.2 $\mathrm{mg} / \mathrm{dL}$ and $6.4 \mathrm{mg} / \mathrm{dL}$ in the counterpulse mode, respectively. However, the normalized index of hemolysis was not different between the continuous and co-pulse modes ( 0.000944 vs 0.000843 , respectively). In addition, we found focal myocardial fibrosis (Figure 4, $A$ and $C$ ), but this was not affected by LVAD implantation. No remarkable change was found in the cardiomyocyte size after support with this system, although the mode was switched in each goat (Figure $4, B$ and $D$ ).

\section{DISCUSSION}

For the first time, we have reported an evaluation of the effects of the NHLCS in a chronic heart failure model. To our knowledge, several reports have evaluated the effects of a control system with electrocardiogram-synchronized RS changes. ${ }^{8,10,12,14-16}$ However, our study is the first to evaluate this system in a conscious animal model of chronic heart failure. Our results have indicated the feasibility of the long-term use of this system. Thus, our results provide information regarding what might be expected when this system is used in the clinical setting. 


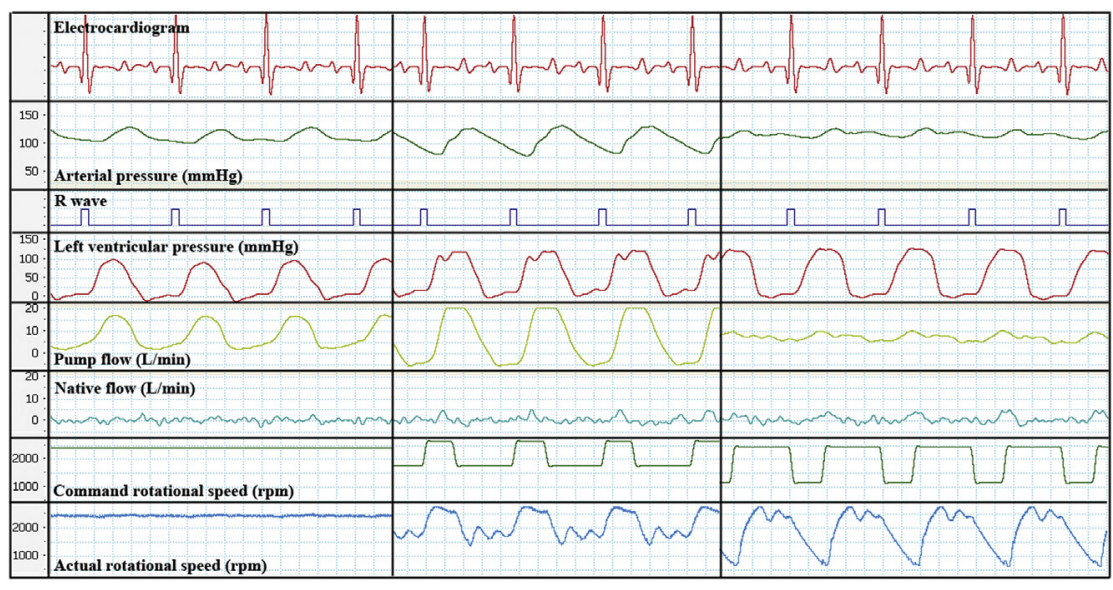

Continuous mode

Co-pulse mode

Counter-pulse mode

FIGURE 1. Sample pressure and flow waveforms at full bypass in each mode. In the co-pulse mode, the pump flow increased in the systolic phase, which was followed by an increase in the rotational speed (RS). In contrast, in the counterpulse mode, the pump flow had increased in the diastolic phase, followed by an increase in the RS compared with the continuous mode.

Furthermore, the results showed no adverse events such as an increase of hemolysis or myocardial overload, and the system provided reliable support.

According to a previous report, unloading of the left ventricle is a factor that promotes cardiac recovery. ${ }^{4}$ However, we have assumed that unloading is not the only factor that promotes cardiac functional recovery. Excessive unloading of the left ventricle by an LVAD could lead to cardiac disuse atrophy. ${ }^{6}$ Furthermore, clenbuterol, a $\beta_{2}$-receptor agonist, has been reported to increase the success rate of BTR. ${ }^{17,18}$ From these findings, a favorable cardiac workload for the native heart according to the patient's condition should be considered to achieve optimal cardiac recovery. Because the co-pulse mode can provide active LV load management, the native heart can contract by itself in this mode; therefore, it acts as the cardiac training mode. The counterpulse mode can decrease the LV load. In the counterpulse mode, the native heart can rest, but systemic assistance is maintained. Therefore, it can be used as a cardiac resting mode.

Umeki and colleagues ${ }^{10,12}$ demonstrated the ability of the newly developed NHLCS to control the LVEDV and myocardial oxygen consumption. These parameters were increased in the co-pulse mode and decreased in the counterpulse mode in those studies. Our results were largely similar to theirs, although several differences were present. First, we required a higher RS speed to achieve the target bypass rate, because the mean blood pressure was greater

TABLE 1. Hemodynamic data

\begin{tabular}{|c|c|c|c|c|c|c|c|c|c|}
\hline \multirow[b]{2}{*}{ Variable } & \multicolumn{3}{|c|}{$\mathbf{5 0} \%$ Bypass rate } & \multicolumn{3}{|c|}{$75 \%$ Bypass rate } & \multicolumn{3}{|c|}{$100 \%$ Bypass rate } \\
\hline & $\begin{array}{l}\text { Continuous } \\
\text { mode }\end{array}$ & $\begin{array}{l}\text { Co-pulse } \\
\text { mode }\end{array}$ & $\begin{array}{c}\text { Counterpulse } \\
\text { mode }\end{array}$ & $\begin{array}{l}\text { Continuous } \\
\text { mode }\end{array}$ & $\begin{array}{l}\text { Co-pulse } \\
\text { mode }\end{array}$ & $\begin{array}{c}\text { Counterpulse } \\
\text { mode }\end{array}$ & $\begin{array}{l}\text { Continuous } \\
\text { mode }\end{array}$ & $\begin{array}{l}\text { Co-pulse } \\
\text { mode }\end{array}$ & $\begin{array}{c}\text { Counterpulse } \\
\text { mode }\end{array}$ \\
\hline $\begin{array}{l}\text { HR (beats/ } \\
\text { min) }\end{array}$ & $126.0 \pm 16.4$ & $128.7 \pm 16.5$ & $126.0 \pm 15.7$ & $128.5 \pm 16.8$ & $128.8 \pm 19.7$ & $127.6 \pm 18.1$ & $126.0 \pm 15.6$ & $128.7 \pm 16.5$ & $126.8 \pm 16.4$ \\
\hline $\begin{array}{l}\text { Mean CVP } \\
(\mathrm{mm} \mathrm{Hg})\end{array}$ & $-3.2 \pm 2.9$ & $-3.0 \pm 2.8$ & $-2.1 \pm 3.2$ & $-3.4 \pm 3.4$ & $-2.8 \pm 4.2$ & $-3.8 \pm 4.3$ & $-2.5 \pm 3.2$ & $-1.6 \pm 3.3$ & $-3.3 \pm 3.9$ \\
\hline $\begin{array}{l}\text { Mean AoP } \\
(\mathrm{mm} \mathrm{Hg})\end{array}$ & $87.2 \pm 21.3$ & $83.7 \pm 19.1$ & $87.0 \pm 20.6$ & $87.8 \pm 18.2$ & $86.4 \pm 20.3$ & $92.0 \pm 21.0$ & $94.4 \pm 20.0$ & $91.2 \pm 19.9$ & $95.0 \pm 20.2$ \\
\hline $\mathrm{PF}(\mathrm{L} / \mathrm{min})$ & $2.0 \pm 0.6^{*}, \dagger$ & $2.1 \pm 0.6^{*}, \dagger$ & $2.0 \pm 0.6^{*}, \dagger$ & $3.3 \pm 1.2 \dagger, \ddagger$ & $3.2 \pm 1.0 \dagger, \ddagger$ & $3.2 \pm 0.8 \dagger, \ddagger$ & $4.3 \pm 1.1^{*}, \ddagger$ & $4.3 \pm 1.4^{*}, \ddagger$ & $4.2 \pm 1.0^{*}, \frac{\dagger}{\ddagger}$ \\
\hline $\begin{array}{l}\text { Total flow } \\
\text { (L/min) }\end{array}$ & $3.9 \pm 1.3$ & $3.9 \pm 1.3$ & $3.9 \pm 1.3$ & $4.3 \pm 1.5$ & $4.3 \pm 1.3$ & $4.2 \pm 1.2$ & $4.1 \pm 1.2$ & $4.0 \pm 1.4$ & $4.0 \pm 1.3$ \\
\hline $\mathrm{RS}$ (rpm) & $1648 \pm 116^{*}, \dagger$ & $1721 \pm 295^{*}, \dagger$ & $1563 \pm 158^{*}, \dagger$ & $1890 \pm 134 \dagger, \ddagger, \S$ & $1852 \pm 177 \dagger, \dagger, \S$ & $1637 \pm 164 \dagger, \dagger, \|, \Phi$ & $2046 \pm 102^{*}, \dagger, \S$ & $1941 \pm 276^{*}, \ddagger$ & $1783 \pm 204^{*}, \ddagger$ \\
\hline $\begin{array}{l}\text { Bypass } \\
\quad \text { rate }(\%)\end{array}$ & $51.2 \pm 4.3^{*}, \dagger$ & $53.3 \pm 4.5^{*}, \dagger$ & $52.6 \pm 4.0^{*}, \dagger$ & $75.0 \pm 3.2 \dagger$ & $76.3 \pm 2.7 \dagger, \ddagger$ & $75.5 \pm 5.6 \dagger, \ddagger$ & $100.0 \pm 4 \dagger, \ddagger$ & $101.6 \pm 9.3^{*}, \ddagger$ & $96.9 \pm 8.7^{*}, \ddagger$ \\
\hline $\begin{array}{l}\text { LVEDP } \\
\qquad(\mathrm{mm} \mathrm{Hg})\end{array}$ & $14.7 \pm 9.3$ & $15.5 \pm 9.9$ & $11.8 \pm 9.5$ & $9.3 \pm 7.9$ & $15.3 \pm 9.1$ & $7.6 \pm 8.6$ & $7.7 \pm 7.7$ & $10.9 \pm 8.5$ & $7.2 \pm 7.8$ \\
\hline
\end{tabular}

$H R$, Heart rate; $C V P$, central venous pressure; $A o P$, aortic pressure; $P F$, pump flow; $R S$, rotational speed; $L V E D P$, left ventricular end-diastolic pressure. $*$ Compared with $75 \%$ with the same mode. $\dagger$ Compared with $100 \%$ with the same mode. $\ddagger$ Compared with $50 \%$ with the same mode. $\S$ Compared with counterpulse mode. ||$C o m p a r e d ~ w i t h ~ c o n t i n u o u s$ mode. $\uparrow$ Compared with co-pulse mode. 

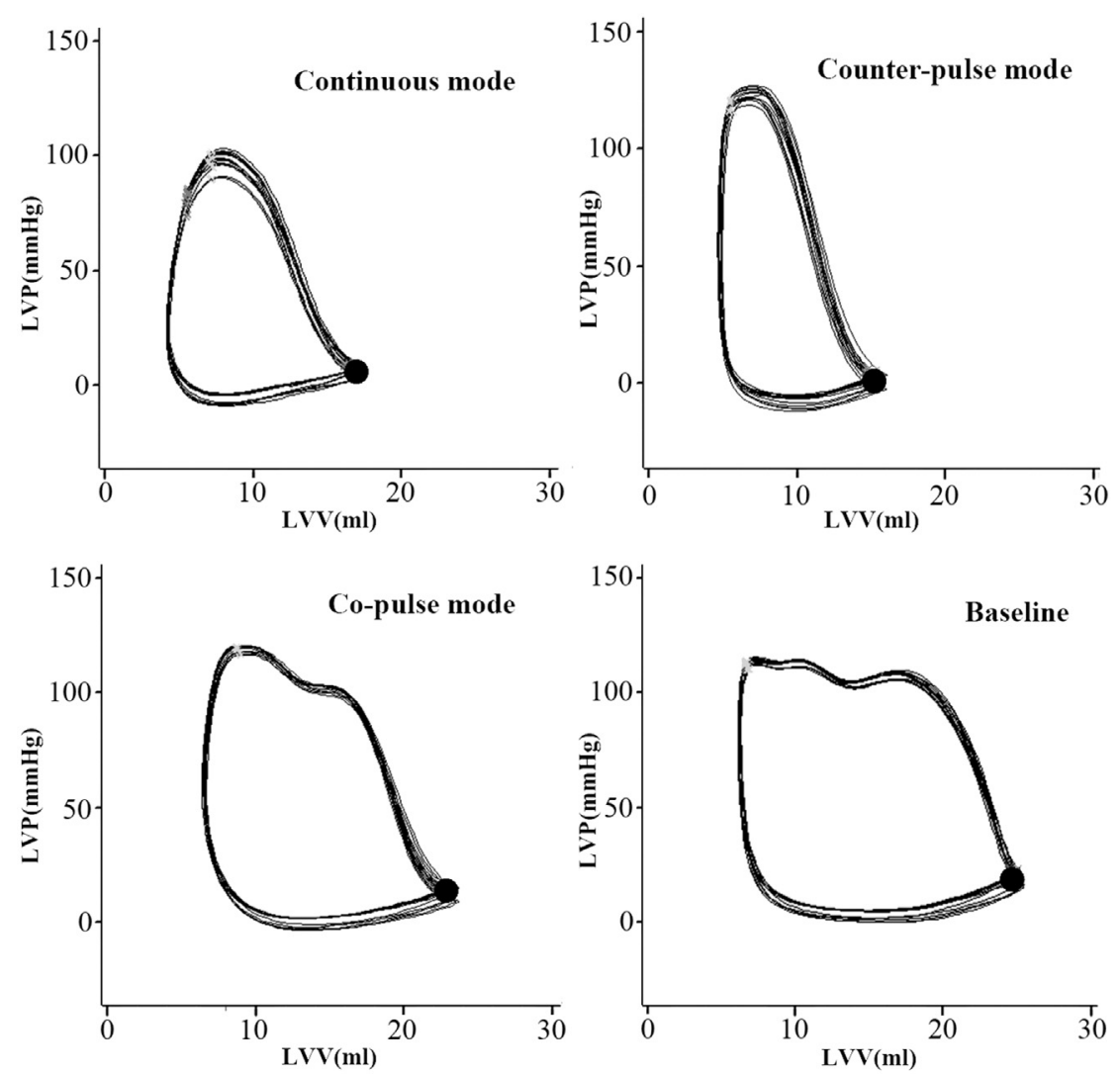

FIGURE 2. Pressure-volume loops at full bypass at baseline and for each mode. The end-diastolic point is shown by the black dot. In the co-pulse mode, the end-diastolic points had shifted rightward compared with those in the continuous mode and had a value that was similar to baseline. In the counterpulse mode, the end-diastolic points had shifted leftward and decreased compared with those in the continuous mode. Furthermore, the pressure-volume loop area was increased in the co-pulse mode and decreased in the counterpulse mode compared with that in the continuous mode. $L V V$, Left ventricular volume; $L V P$, left ventricular pressure.

in the conscious state than in the anesthetized state. Second, we simply compared the effects of the different NHLCS modes in the present study, because we were not able to clamp the LVAD circuit to hold the PF constant. Third, we have demonstrated the effects of NHLCS in a chronic heart failure model.

End-diastolic volume (\%)

Stroke work (\%)
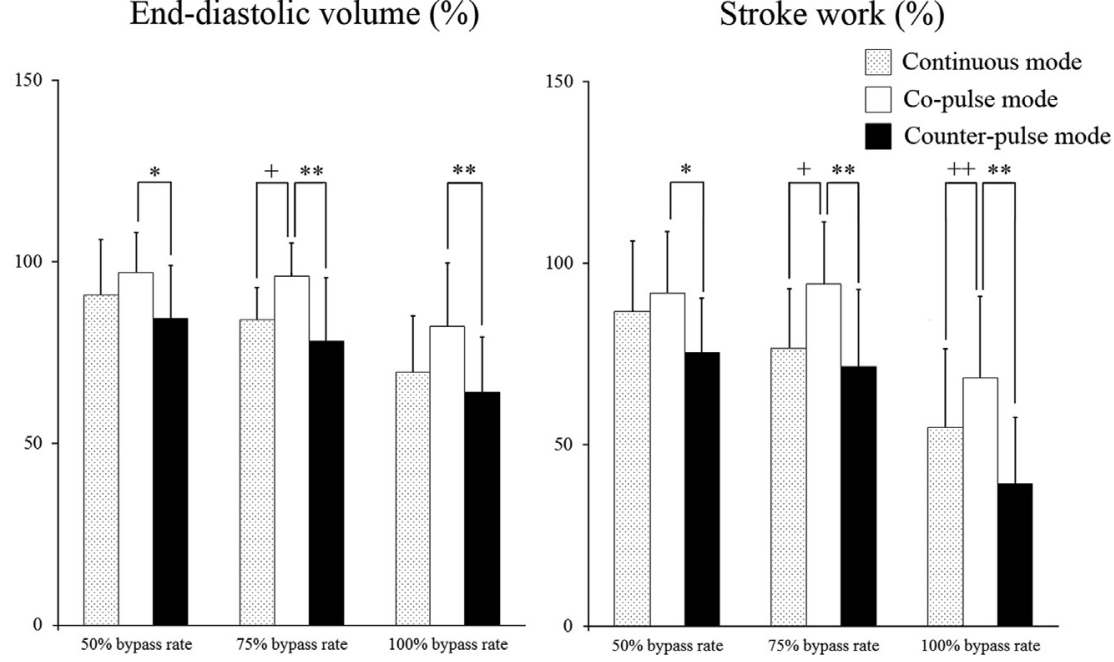

FIGURE 3. The left ventricular end-diastolic volume (LVEDV) and stroke work (SW) are shown relative to the baseline, which was obtained under $0 \%$ bypass $(100 \%)$. A trend was seen for the percentage of LVEDV in the co-pulse mode to increase at each bypass rate and for the LVEDV in the counterpulse mode to decrease compared with that in the continuous mode. In addition, a similar trend was seen for the percentage of SW in the co-pulse mode to increase at each bypass rate and for the $\mathrm{SW}$ in the counterpulse mode to decrease compared with that in the continuous mode. ${ }^{*},+P<.05 ;++,{ }^{* *} P<.01$. 

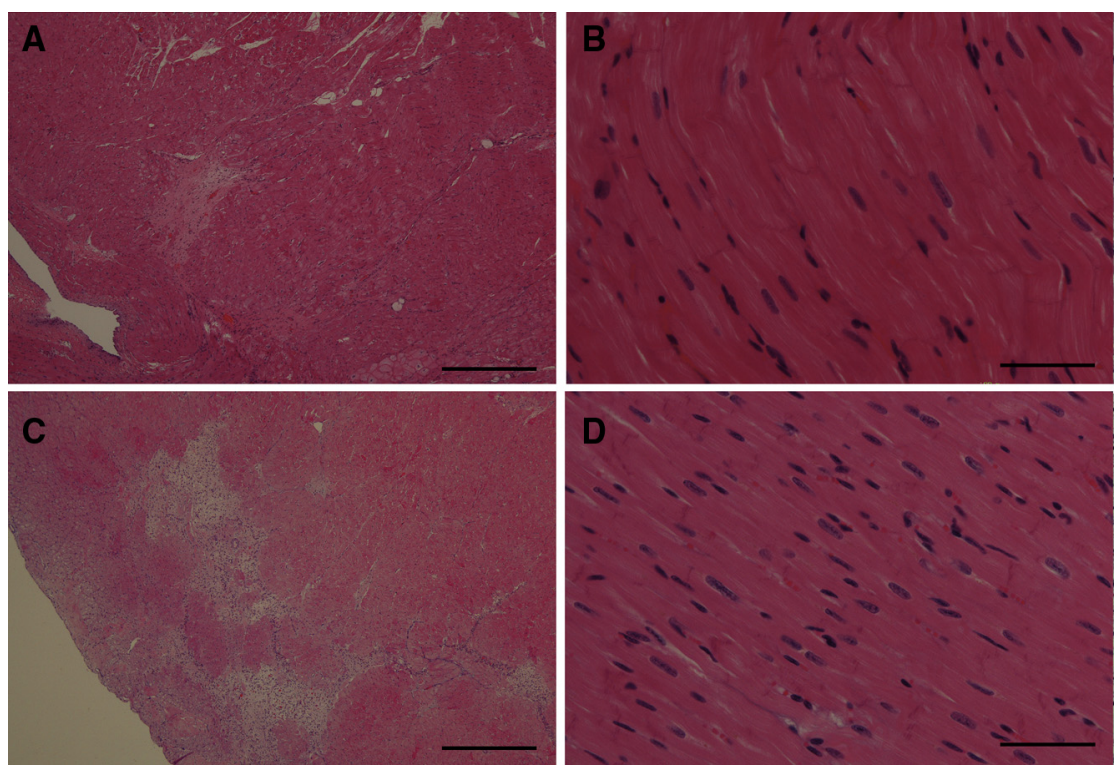

FIGURE 4. Myocardial hematoxylin and eosin stain, (A and B) before, and (C and D) after left ventricular assist device implantation. Scale bars represent (A and C) $500 \mu \mathrm{m}$ and (B and D) $50 \mu \mathrm{m}$.

Recently, it has become clear that electrocardiogramsynchronized RS control systems have beneficial effects on cardiac load and hemodynamics. Significant controversy has resulted regarding the differences between pulsatile and nonpulsatile hemodynamics. ${ }^{19-23}$ Our system can generate artificial pulsatility, in addition to the beneficial effects on the cardiac workload. ${ }^{8}$ In addition, Kishimoto and colleagues $^{24}$ reported that speed modulation can open the aortic valve and might prevent aortic insufficiency. Furthermore, Ando and colleagues ${ }^{25,26}$ reported that to prevent backward flow, it is possible to increase the RS in the diastolic phase and thus create a circuit clamp condition resembling the "off-test mode." We believe that the NHLCS has the potential to improve the success rate of cardiac recovery and allow more options in the treatment of patients with an implanted rotary blood pump.

Our study had several limitations. We used an animal model of LV dysfunction that showed decreased contractility. However, the EF did not actually decrease to a level that would warrant LVAD implantation in a clinical setting. To compare the effect of different NHLCS operating modes, it would be better to use an impaired cardiac function model. ${ }^{10}$ In addition, we did not show actual myocardial recovery, because we tested the NHLCS for 12 hours, which was not long enough to evaluate recovery. However, we have developed a program for the NHLCS that will make it suitable for long-term use. We are currently investigating the actual long-term effects of this system on cardiac recovery and adverse events in a chronic heart failure model.

\section{CONCLUSIONS}

We have shown that the NHLCS can actively control the LV load in a chronic heart failure model. The NHLCS can provide a favorable LV load in each phase of cardiac recovery, contributing to an increase in the success rate of BTR therapy.

The authors are grateful to Dr Daisuke Ogawa in the Sun Medical Technology Research Corporation for creating the programs.

\section{References}

1. Slaughter MS, Rogers JG, Milano CA, Russell SD, Conte JV, Feldman D, et al Advanced heart failure treated with continuous-flow left ventricular assist device. N Engl J Med. 2009;361:2241-51.

2. Starling RC, Naka Y, Boyle AJ, Gonzalez-Stawinski G, John R, Jorde U, et al. Results of the post-US Food and Drug Administration-approval study with a continuous flow left ventricular assist device as a bridge to heart transplantation: a prospective study using the INTERMACS (Interagency Registry for Mechanically Assisted Circulatory Support). J Am Coll Cardiol. 2011;57:1890-8.

3. Kitamura S. Heart transplantation in Japan: a critical appraisal for the results and future prospects. Gen Thorac Cardiovasc Surg. 2012;60:639-44.

4. Frazier OH, Benedict CR, Radovancevic B, Bick RJ, Capek P, Springer WE, et al. Improved left ventricular function after chronic left ventricular unloading. Ann Thorac Surg. 1996;62:675-81.

5. Maybaum S, Mancini D, Xydas S, Starling RC, Aaronson K, Pagani FD, et al. Cardiac improvement during mechanical circulatory support: a prospective multicenter study of the LVAD working group. Circulation. 2007;115:2497-505.

6. Kinoshita M, Takano H, Taenaka Y, Mori H, Takaichi S, Noda H, et al. Cardiac disuse atrophy during LVAD pumping. ASAIO Trans. 1988;34:208-12.

7. Yamazaki K, Kihara S, Akimoto T, Tagusari O, Kawai A, Umezu M, et al. EVAHEART: an implantable centrifugal blood pump for long-term circulatory support. Jpn J Thorac Cardiovasc Surg. 2002;50:461-5.

8. Ando M, Nishimura T, Takewa Y, Yamazaki K, Kyo S, Ono M, et al. Electrocardiogram-synchronized rotational speed change mode in rotary pumps could improve pulsatility. Artif Organs. 2011;35:941-7.

9. Ando M, Nishimura T, Takewa Y, Ogawa D, Yamazaki K, Kashiwa K, et al. A novel counterpulse drive mode of continuous-flow left ventricular assist devices 
can minimize intracircuit backward flow during pump weaning. J Artif Organs. 2011;14:74-9.

10. Umeki A, Nishimura T, Ando M, Takewa Y, Yamazaki K, Kyo S, et al. Alteration of LV end-diastolic volume by controlling the power of the continuous-flow LVAD, so it is synchronized with cardiac beat: development of a native heart load control system (NHLCS). J Artif Organs. 2012;15:128-33.

11. Dixon JA, Spinale FG. Large animal models of heart failure: a critical link in the translation of basic science to clinical practice. Circ Heart Fail. 2009;2: 262-71.

12. Umeki A, Nishimura T, Takewa Y, Ando M, Arakawa M, Kishimoto Y, et al. Change in myocardial oxygen consumption employing continuous-flow LVAD with cardiac beat synchronizing system, in acute ischemic heart failure models. J Artif Organs. 2013;16:119-28.

13. Kono S, Nishimura K, Yamada T, Oonishi T, Tsukiya T, Akamatsu T, et al. In vivo and in vitro evaluation of the pulsatile mode of a magnetically suspended centrifugal pump. ASAIO J. 1997;43:M580-4.

14. Ando M, Takewa Y, Nishimura T, Yamazaki K, Kyo S, Ono M, et al. A novel counterpulsation mode of rotary left ventricular assist devices can enhance myocardial perfusion. J Artif Organs. 2011;14:185-91.

15. Pirbodaghi T, Axiak S, Weber A, Gempp T, Vandenberghe S. Pulsatile control of rotary blood pumps: does the modulation waveform matter? J Thorac Cardiovasc Surg. 2012;144:970-7.

16. Pirbodaghi T, Weber A, Axiak S, Carrel T, Vandenberghe S. Asymmetric speed modulation of a rotary blood pump affects ventricular unloading. Eur J Cardiothorac Surg. 2013;43:383-8.

17. Hon JK, Yacoub MH. Bridge to recovery with the use of left ventricular assist device and clenbuterol. Ann Thorac Surg. 2003;75:S36-41.

18. Soppa GK, Lee J, Stagg MA, Felkin LE, Barton PJ, Siedlecka U, et al. Role and possible mechanisms of clenbuterol in enhancing reverse remodelling during mechanical unloading in murine heart failure. Cardiovasc Res. 2008;77: 695-6.

19. Nishimura T, Takewa Y, Huang Y, Shirota K, Ramanathan I, Hunyor S. Influence of varying conduit resistance on native heart function with nonpulsatile left heart bypass. J Artif Organs. 2001;4:126-30.

20. Nishimura T, Tatsumi E, Nishinaka T, Taenaka Y, Masuzawa T, Nakata M, et al. Diminished vasoconstrictive function caused by long-term nonpulsatile left heart bypass. Artif Organs. 1999;23:722-6.

21. Nishimura T, Tatsumi E, Nishinaka T, Taenaka Y, Nakata M, Takano H. Prolonged nonpulsatile left heart bypass diminishes vascular contractility. Int J Artif Organs. 1999;22:492-8.

22. Nishimura T, Tatsumi E, Taenaka Y, Nishinaka T, Nakatani T, Masuzawa T, et al. Effects of long-term nonpulsatile left heart bypass on the mechanical properties of the aortic wall. ASAIO J. 1999;45:455-9.

23. Nishinaka T, Tatsumi E, Nishimura T, Shioya K, Ohnishi H, Taenaka Y, et al. Change in vasoconstrictive function during prolonged nonpulsatile left heart bypass. Artif Organs. 2001;25:371-5.

24. Kishimoto Y, Takewa Y, Arakawa M, Umeki A, Ando M, Nishimura T, et al. Development of a novel drive mode to prevent aortic insufficiency during continuous-flow LVAD support by synchronizing rotational speed with heartbeat. J Artif Organs 2013;129-37.

25. Ando M, Nishimura T, Takewa Y, Ogawa D, Yamazaki K, Kashiwa K, et al. What is the ideal off-test trial for continuous-flow ventricular-assist-device explantation? Intracircuit back-flow analysis in a mock circulation model. J Artif Organs. 2011;14:70-3.

26. Ando M, Nishimura T, Takewa Y, Kyo S, Ono M, Taenaka Y, et al. Creating an ideal "off-test mode" for rotary left ventricular assist devices: establishing a safe and appropriate weaning protocol after myocardial recovery. $J$ Thorac Cardiovasc Surg. 2012;143:1176-82. 